score. CIBERSORT algorithm analysis showed that the two common TIICs in PDAC tissues were macrophages and $\mathrm{T}$ lymphocytes. The proportions of M2 macrophages were significantly higher in P311 high group compared with P311 low group $(\mathrm{P}<0.001)$. Additionally, a positive association was shown between P311 mRNA expression and transcript levels of IL10 and CSF-1 in PDAC. Moreover, the percentages of CD68/ CD163 double-positive cells indicative of M2 macrophages were higher in PMA-stimulated THP-1 cells cocultured with the conditioned medium obtained from P311 overexpressed PANC-1 cells than those from vector control cells.

Conclusions Collectively, our studies provide evidence for a novel role for P311 in M2 macrophage polarization and infiltration, uncovering the underlying oncogenic role of P311 in PDAC. Targeting P311 may serve as a novel potential therapeutic option to reset TAM polarization toward an antitumor state in PDAC.

\section{IDDF2021-ABS-0074 GUT MICROBIAL METABOLITE OF TRYPTOPHAN RETARDS INTESTINAL STEM CELL MAINTENANCE UNDER PSYCHOLOGICAL STRESS IN MICE}

${ }^{1}$ Xiao Zheng*, ${ }^{2}$ Yali Liu. ${ }^{1}$ China Pharmaceutical University, China; ${ }^{2}$ Chinses University of Hongkong, China

10.1136/gutjnl-2021-IDDF.31
Background Psychosocial stress has a negative impact on gastrointestinal homeostasis in part via brain-gut microbiota mechanisms that are poorly understood. Despite the significant association of intestinal stem cell (ISC) function with gut

A

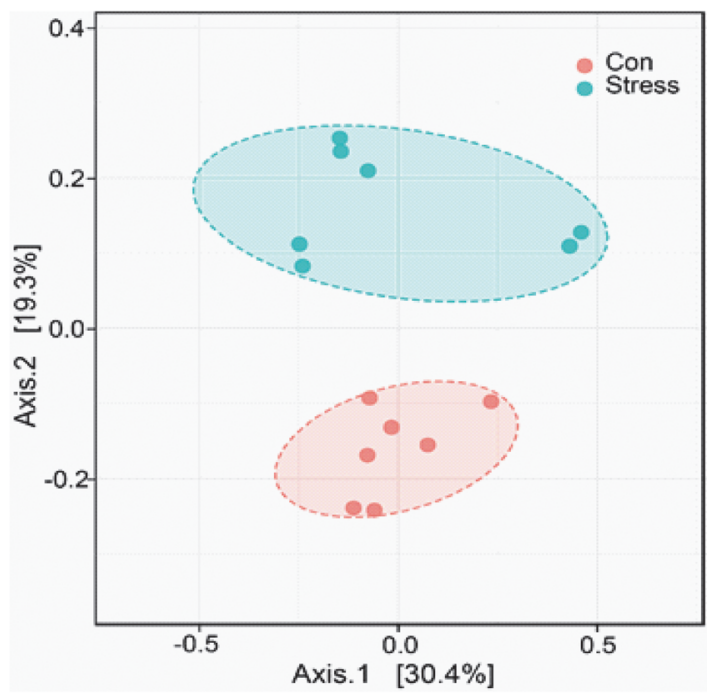

Abstract IDDF2021-ABS-0074 Figure 1
B

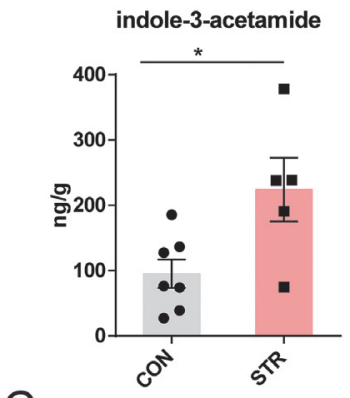

C
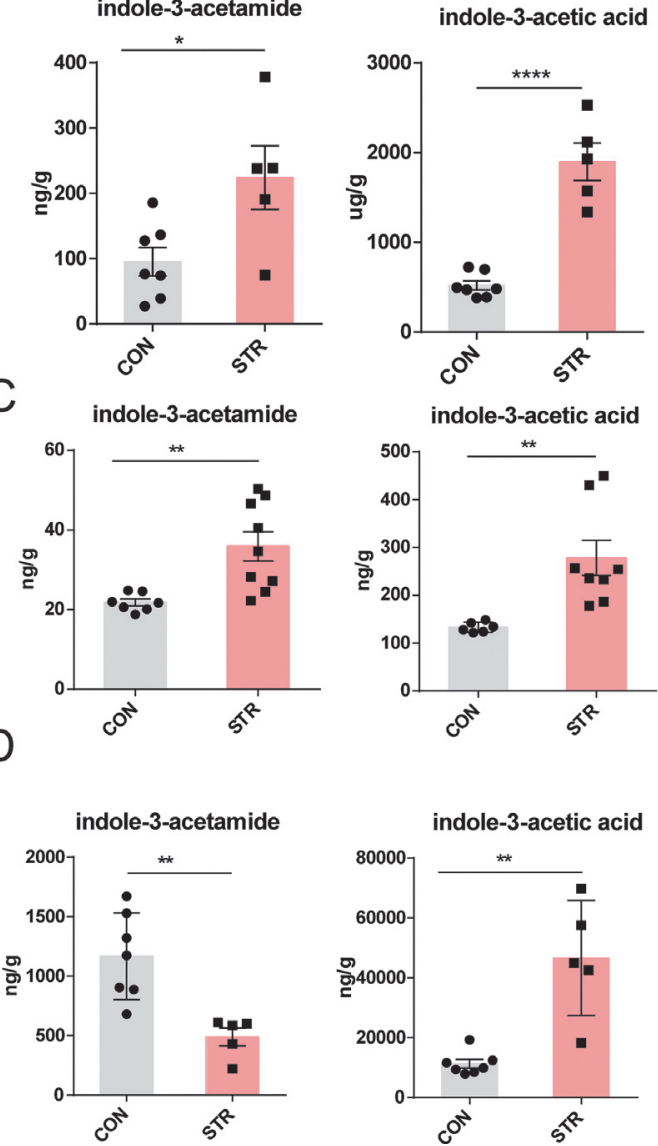
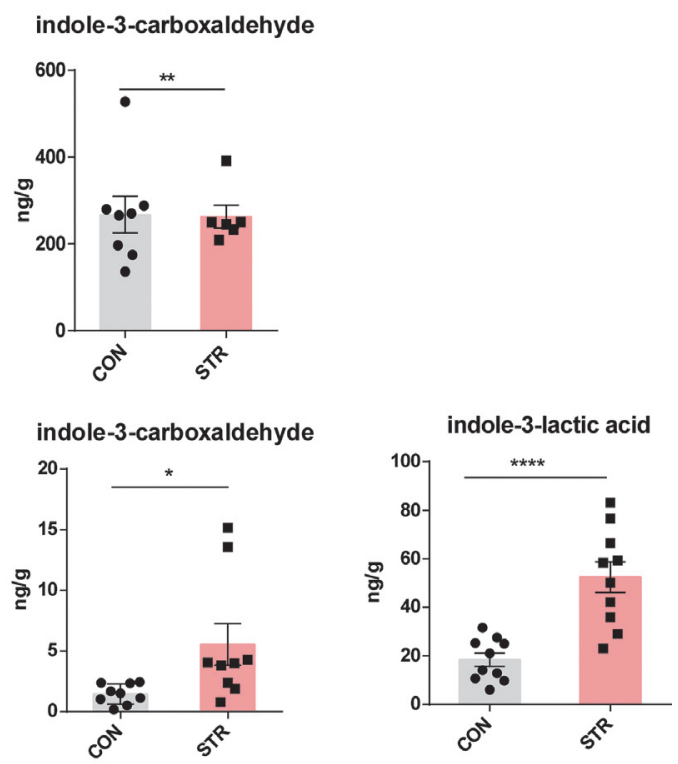

Abstract IDDF2021-ABS-0074 Figure 2 

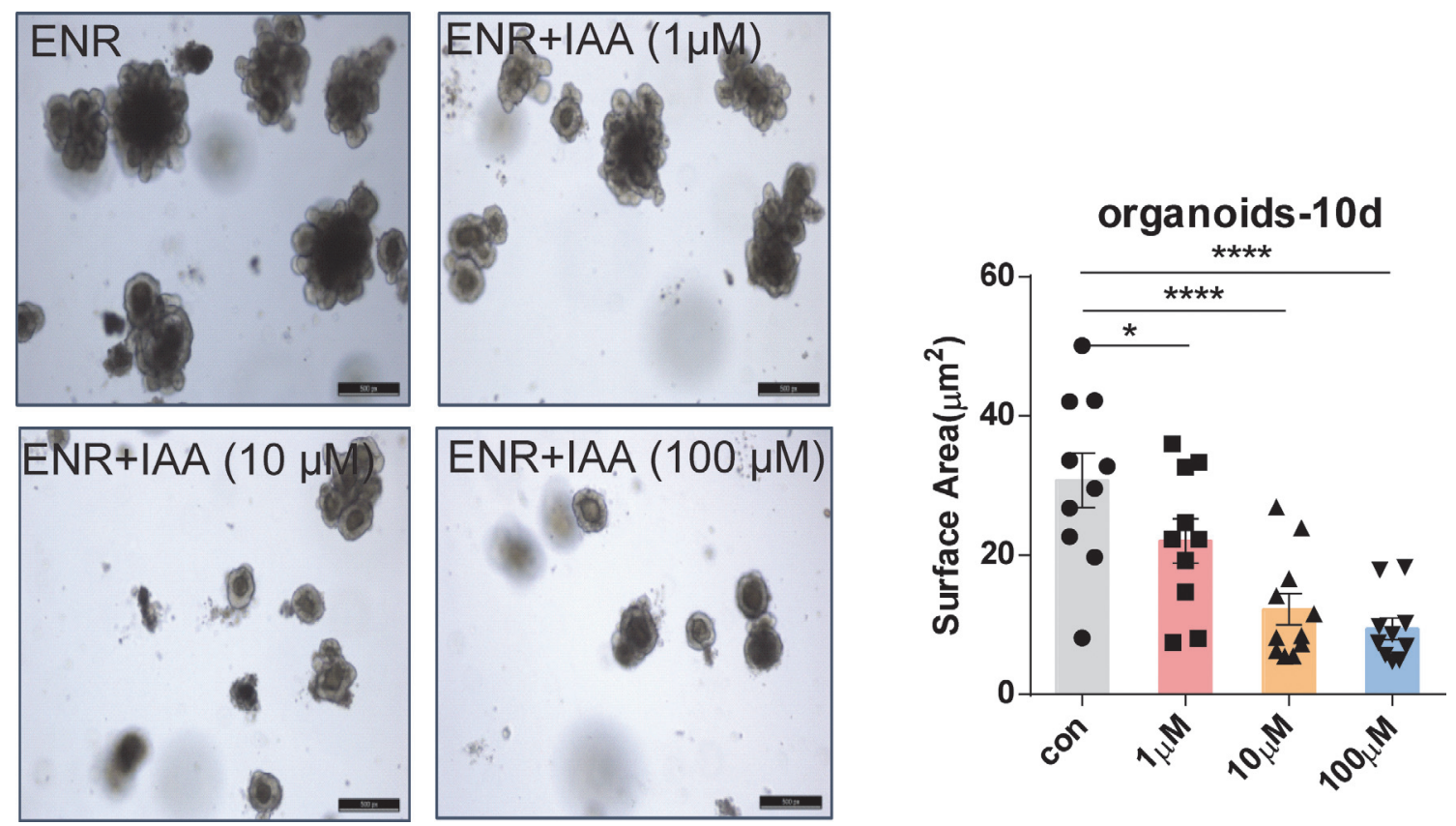

Abstract IDDF2021-ABS-0074 Figure 3

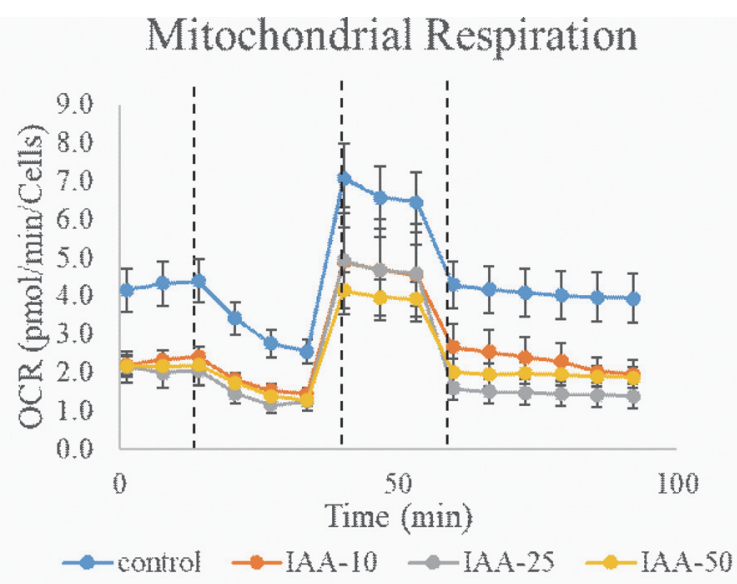

Abstract IDDF2021-ABS-0074 Figure 4

homeostasis, the contribution of ISCs in chronic psychological stress to disrupt the intestinal system remains poorly defined. Methods A chronic restraint stress (CRS) model was established to simulate psychological stress. The number of small intestinal crypt proliferating cells and intestinal epithelial cells including goblet cells and Paneth cells were measured by immunofluorescence staining to evaluate the ISC proliferation and differentiation. Isolated intestinal crypt stem cells from CRS model mice and control mice were cultured in vitro to evaluate the functional status of ISCs. The mitochondrial OXPHOS ability of intestinal crypt stem cells in CRS mice and control mice was assessed using Seahorse mitochondrial stress test. 16S rRNA analysis was used to investigate the disturbance of the intestinal flora in mice under CRS condition. Antibiotic intervention was used to determine the effect of intestinal flora on the damage of ISCs induced by CRS. Quantitative analysis of microbe-derived indole metabolites in the feces, ileum and colon was performed in CRS mice with or without antibiotic intervention. Differential gene enrichment analysis was performed to find the main signal pathway regulated by indole-3-acetic acid (IAA).

Results Here we show that chronic restraint stress in mice alters gut microbiota structure and increases IAA to disrupt ISC maintenance (IDDF2021-ABS-0074 Figure 1, IDDF2021ABS-0074 Figure 2, IDDF2021-ABS-0074 Figure 3, IDDF2021-ABS-0074 Figure 4). Antibiotic rescues, while IAA derivative pre-treatment retards, ISC-mediated epithelial repair under stress (IDDF2021-ABS-0074 Figure 5). Mechanistically,

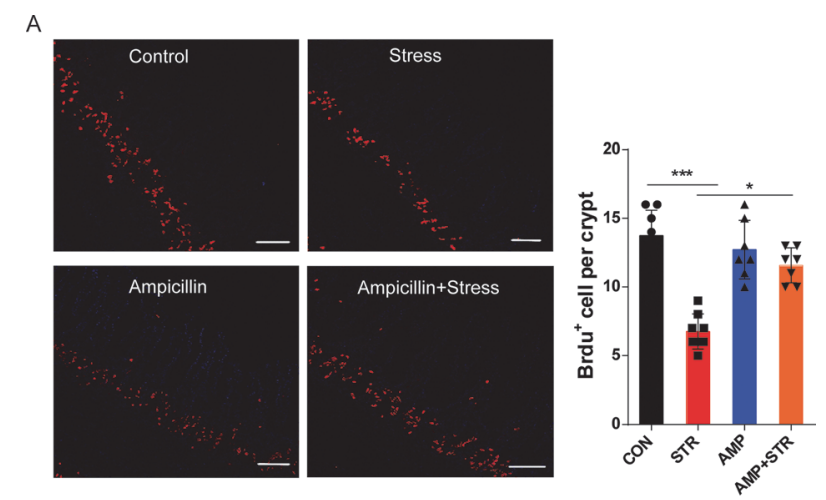

B

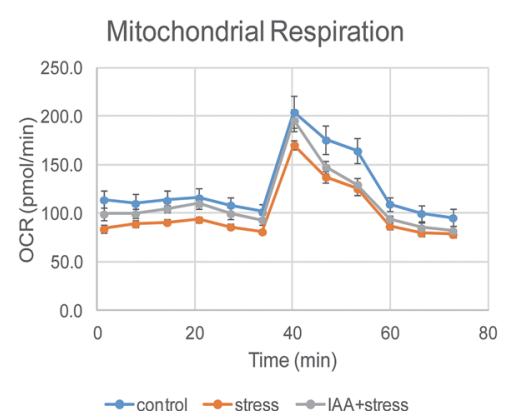

Abstract IDDF2021-ABS-0074 Figure 5 
A

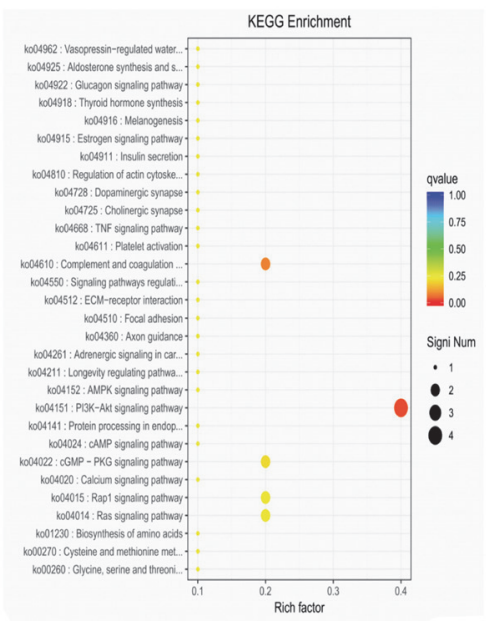

Abstract IDDF2021-ABS-0074 Figure 6

C
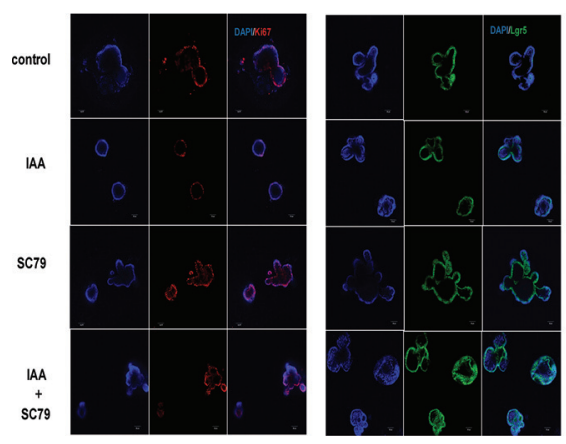

B

Mitochondrial Respiration

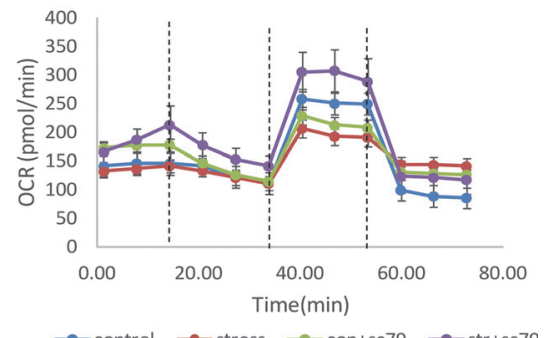

D
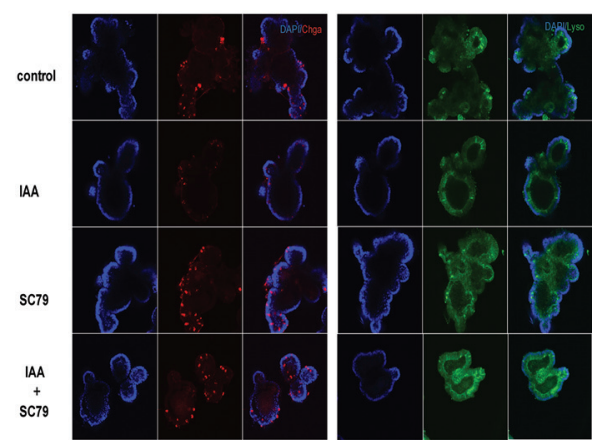

Abstract IDDF2021-ABS-0074 Figure 7

A

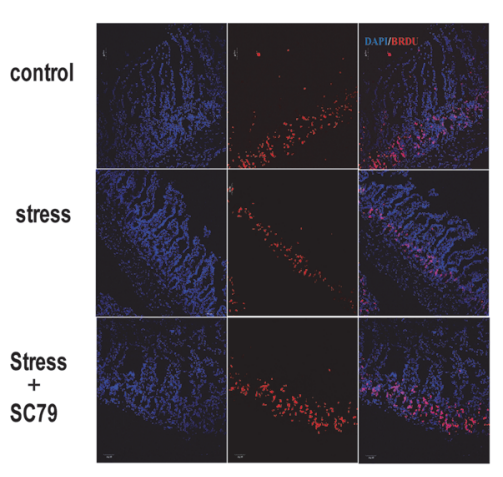

B

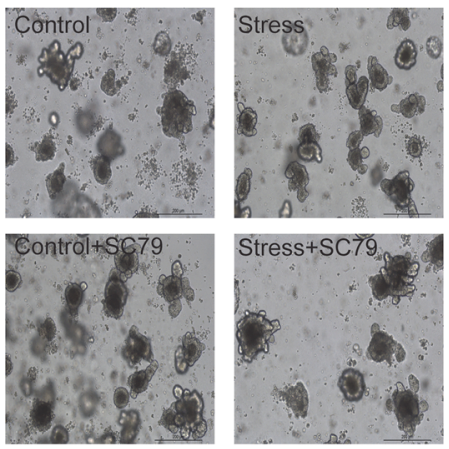

C

D
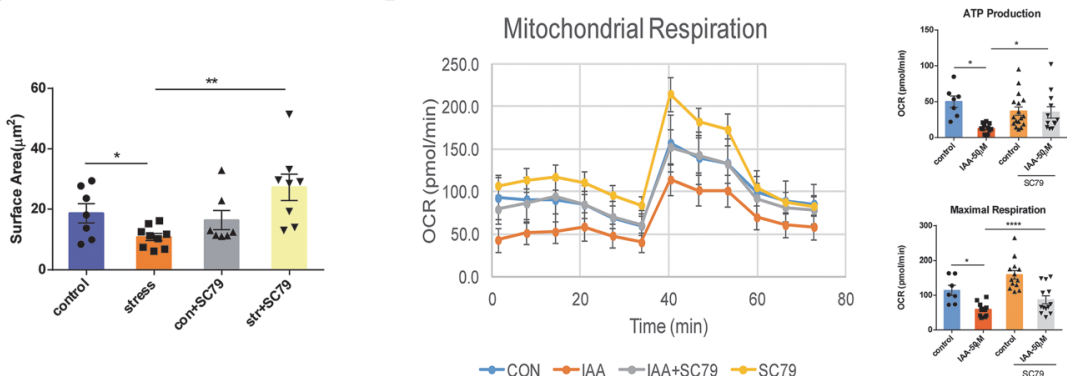
IAA disrupts mitochondrial oxidative phosphorylation program via the Akt/mTOR axis, thereby suppressing the differentiation of ISC and organoid formation (IDDF2021-ABS-0074 Figure 6, IDDF2021-ABS-0074 Figure 7). Activation of Akt reverses IAA-induced impairment of mitochondrial energetics, stem cell maintenance and epithelial renewal in chronically-stressed mice (IDDF2021-ABS-0074 Figure 8).

Conclusions Our results connect gut dysbiosis and IAA to ISC dysfunction during psychological stress and suggest that modulating gut microbial metabolism may prevent the gut from the deleterious effect of stressful events.

\section{IDDF2021-ABS-0076 LNCRNA TNFRSF10A-AS1 MEDIATES TUMORIGENESIS OF GASTRIC CANCER BY DIRECTLY BINDING TO MPZL1 AND ASSOCIATES WITH PATIENT OUTCOMES}

${ }^{1}$ Donglei Sun*, ${ }^{2}$ Hongyan Gou, ${ }^{1}$ Dandan Wang, ${ }^{3} J u n$ Yu. ${ }^{1}$ The Second Hospital of Hebei Medical University, China; ${ }^{2}$ CUHK Shenzhen Research Institute, The Chinese University of Hong Kong, China; ${ }^{3}$ The Chinese University of Hong Kong, Hong Kong, China

\subsection{6/gutjn--2021-IDDF.32}

Background LncRNA was known to be closely associated with the progression of human tumors. The role of new IncRNA TNFRSF10A-AS1 in the pathogenesis and progression of gastric tumor is still unclear. The aim of this study was to investigate the function of TNFRSF10A-AS1 and the underlying mechanism in the pathogenesis and progression of gastric cancer.

Methods The clinical impact of TNFRSF10A-AS1 was assessed in 105 patients with gastric cancer. The biological function of TNFRSF10A-AS1 was studied in vitro and in vivo. TNFRSF10A-AS1 downstream effector were identified by RNA FISH, RNA sequencing, RNA pulldown and rescue assay.

Results TNFRSF10A-AS1was upregulated in gastric cancer cell lines and tissues. Multivariate analysis showed that gastric cancer patients with TNFRSF10A-AS1 overexpression had a significantly shortened survival. TNFRSF10A-AS1 significantly promoted gastric cancer cell proliferation, cell-cycle transformation, and migration/invasion, but suppressed cell apoptosis. Silencing TNFRSF10A-AS1 expression exerted opposite effects in vitro and significantly inhibited xenograft tumor growth in nude mice. Mechanically, TNFRSF10A-AS1 directly bound to MPZL1 and activated MPZL1 transcription. Knockdown MPZL1 abrogated the effect of TNFRSF10A-AS1 in the tumor-promoting function.

Conclusions TNFRSF10A-AS1 directly binds to oncogenic MPZL1 to induce its expression. TNFRSF10A-AS1 plays a pivotal oncogenic role in gastric carcinogenesis and is an independent prognostic factor for gastric cancer patients.

\section{IDDF2021-ABS-0094 VIRULENCE FACTORS AND RESISTANCE GENES CARRIED DIFFERENCES OF KLEBSIELLA PNEUMONIAE ISOLATED FROM PATIENTS WITH PNEUMONIA AND PYOGENIC LIVER ABSCESS}

Lin Gan*, Chao Yan, Jinghua Cui, Guanhua Xue, Junxia Feng, Hanqing Zhao, Yanling Feng, Jing Yuan. Capital Institute of Pediatrics, China

10.1136/gutjnl-2021-IDDF.33
Background The bacterium Klebsiella pneumoniae of family Enterobacteriacea is a well-known opportunistic pathogen that colonizes intestinal and respiratory tract. While K. pneumoniae is a common cause of nosocomial and community-acquired infections, including diarrhea, pneumonia and pyogenic liver abscess, little is known about the population structure of this bacterium. Thus it is likely that the epidemiological characteristics of $\mathrm{K}$. pneumoniae isolate from carriers and clinic patients, when combined to their genomic information, might provide some insight into pathogenic Klebsiella prevention and control.

Methods Two hundred and thirty-two K. pneumoniae isolates (including 38 isolates from carriers, 124 isolates from pyogenic liver abscess patients and 70 isolates from pneumonia patients) were collected from 9 provinces of China in 20132020. Sequencing was performed on the Illumina Hiseq PE150 platform, and the genome sequences were assembled by SOAP denovo. Multilocus sequence typing (MLST) analysis was done by submitting sequences to the Institute Pasteur K. pneumoniae MLST database. Pan-genome analysis was performed by software Snippy, Gubbins and Roary, and the gene contents were identified by software VFanalyzer, Resfinder and PlasmidFinder.

Results The 232 isolates were subtyped into 74 STs. The isolates from different sources have their own STs, and the predominant subtypes of liver abscess patients and pneumonia patients were ST23 and ST11, respectively. PCA analysis ( $p$ $=0.001)$ on accessory gene content also distinguished the three phylogroups, which are consistent with the source of isolates. The isolates collected from liver abscess patients carried significantly more $(p=0.000)$ virulence factors, and the isolates sourced from pneumonia patients harbored significantly more $(p=0.000)$ resistance genes and replicons. Besides, there was a strong link between Salmonchelin and the isolates sourced from liver abscess patients. Ninety-eight percent isolates of liver abscess strongly correlated STs and only two percent isolates of pneumoniae correlated STs carried Salmonchelin.

Conclusions These data provide genomic support for the proposal that isolates collected from carrier, liver abscess and pneumoniae patients have their distinct genomic features. And the isolates from different sources are largely nonoverlapping.

\section{IDDF2021-ABS-0097 P38 $\alpha$ DEFICIENCY IN MACROPHAGES AMELIORATES MURINE EXPERIMENTAL COLITIS BY REGULATING INFLAMMATION AND IMMUNE PROCESS}

Wei Chen*, Jinshui Zhu, Jing Zhang, Rui Liang, Youcai Yi, Xiaoyu Chen, Huining Fan. Shanghai Jiao Tong University Affiliated Sixth People's Hospital, China

\subsection{6/gutjnl-2021-IDDF.34}

Background $\mathrm{P} 38 \alpha$ is a mitogen-activated protein kinase (MAPK) that mediates inflammatory responses. P38 $\alpha$ alterations have been associated with inflammation-related diseases. However, the role of macrophages-derived p38 $\alpha$ in dextran sulfate sodium (DSS)-induced murine experimental colitis remains unclear. We characterized the role of macrophagesderived $\mathrm{p} 38 \alpha$ in DSS-induced colitis.

Methods The expression of macrophage-derived $\mathrm{p} 38 \alpha$ in human colitis and normal tissues was measured by 